\title{
Unc5D regulates p53-dependent apoptosis in neuroblastoma cells
}

\author{
HONG WANG ${ }^{1}$, QIONG WU ${ }^{2}$, SHUANG LI ${ }^{1}$, BIN ZHANG $^{1}$, ZUOFEI CHI $^{1}$ and LIANGCHUN HAO ${ }^{1}$ \\ ${ }^{1}$ Department of Pediatric Hematology and Oncology, Hematology Center; ${ }^{2}$ Department of Pediatric Neurology, \\ Shengjing Hospital, China Medical University, Shenyang, Liaoning 110004, P.R. China
}

Received July 25, 2013; Accepted February 18, 2014

DOI: $10.3892 / \mathrm{mmr} .2014 .2100$

\begin{abstract}
The mechanism of apoptosis via the p53-dependent pathway remains to be fully understood. In the present study, a novel p53 target gene, Unc5D, was identified and its possible function in human neuroblastoma cells was investigated. The apoptotic effects of Unc5D in SK-N-BE $\left(\mathrm{p}^{-/} 3^{-}\right)$and

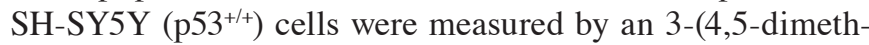
ylthiazol-2-yl)2,5-diphenyltetrazolium bromide solution assay. Reverse transcription-polymerase chain reaction (RT-PCR) was also performed to detect the endogenous expression of Unc5D. In H1299 (p53 ${ }^{-/}$) cells, following overexpression of p53, RT-PCR and western blot analysis were used to detect the Unc5D mRNA and protein levels. In order to detect the promoter activity in the Unc5D gene, a luciferase assay was performed. Finally, to confirm the activate site of p53 subsequent to DNA damage, western blot analysis was used to analyze the phosphorylation site of Unc5D stable and mock clones in H1299 cells by co-expression of p53. Unc5D-induced apoptosis may be largely dependent on the p53 status. Notably, Unc5D was found to be a direct transcriptional target of p53. During adriamycin-mediated apoptosis, Unc5D was significantly induced in p53-proficient SH-SY5Y cells but not in p53-deficient SK-N-BE cells. Overexpression of p53 resulted in an increase in the expression levels of endogenous Unc5D. Additionally, two elements were identified in the sequence of Unc5D. Notably, Unc5D expression also induced phosphorylation of p53 at serine-15. Unc5D is thus a newly identified transcriptional target of pro-apoptotic p53 and may also act upstream of p53 to induce p53-dependent apoptosis by phosphorylation at ser- 15 .
\end{abstract}

\section{Introduction}

Neuroblastoma is the most common type of extracranial solid cancer in children. It is a neuroendocrine tumor, that may arise from any neural crest element of the sympathetic

Correspondence to: Dr Hong Wang, Department of Pediatric Hematology and Oncology, Hematology Center, Shengjing Hospital, China Medical University, 36 Sanhao Street, Shenyang, Liaoning 110004, P.R. China

E-mail: cmu2hwanghong@163.com

Key words: neuroblastoma, Unc5D, apoptosis, p53 nervous system (1). It comprises $6-10 \%$ of all childhood cancers, and $15 \%$ of cancer-related mortalities in children. The annual mortality rates are 10 per million children aged $0-4$ years and 4 per million children aged 4-9 years (2). Although there has been an improvement in the treatment methods, the mortality rate of neuroblastoma remains extremely high. The most frequently mutated gene among all genes known to be involved in human cancer, including human neuroblastoma, is the tumor suppressor p53 (3). p2 $1^{\mathrm{WAF}}$, a cyclin-dependent kinase inhibitor, is known to be the principle mediator of p53 in the apoptotic pathway and has been shown inhibit the cyclin-dependent kinase p53R2 (4-6) and several mitochondrial proteins, including Bax, Noxa and mouse double minute 2 homolog (7-9). The products of these genes have diverse functions, including cell-cycle arrest, apoptosis, DNA repair, angiogenesis and transcription. Apoptosis is hypothesized to be vital in preventing cells from undergoing malignant transformation by eliminating damaged cells (10). A number of studies have indicated that the mitochondrial and the death-receptor apoptotic pathways are significant in this process $(7,8,10)$. However, identification of a number of p53-regulated genes clearly indicated that there are numerous p53-regulated apoptotic genes that are not involved in these two pathways, implying that the mechanism for p53-dependent apoptosis remains unclear.

Unc5B is one of four related receptors for netrin-1 (Unc5A, Unc5B, Unc5C and Unc5D). Notably, Unc5B was directly regulated by p53 (11), providing a novel perspective on the role of the p53-regulatory system in apoptosis. In the present study, Unc5D was demonstrated to be a direct target of the p53 response to DNA damage and possibly form a type of feedback to induce p53-dependent apoptosis by phosphorylation of serin-15.

\section{Materials and methods}

Cell culture and transfection. SK-N-BE $\left(\mathrm{p} 53^{-/}\right)$and SH-SY5Y $\left(\mathrm{p} 53^{+/+}\right)$human neuroblastoma cells, and H1299 (p53 $\left.3^{-/}\right)$ human lung carcinoma cells were maintained in RPMI-1640 medium containing $10 \%$ heat-inactivated fetal bovine serum (Invitrogen Life Technologies, Carlsbad, CA, USA) and penicillin/streptomycin. Cultures were maintained at $37^{\circ} \mathrm{C}$ in a water-saturated atmosphere of $5 \% \mathrm{CO}_{2}$ in air for transfection. The pCDNA3.1-p53 plasmid was transfected into H1299 cells in a dose- and time-dependent manner with the indicated combination expressing plasmid using Lipofectamine ${ }^{\text {TM }} 2000$ transfection reagent according to the manufacturer's 
instructions (Invitrogen Life Technologies).

3-(4,5-dimethylthiazol-2-yl)2,5-diphenyltetrazolium bromide solution (MTT) assay. The SH-SY5Y and SK-N-BE cells were seeded in 96 -well culture plates as $5 \times 10^{3} /$ well and were allowed to attach overnight. The cells were treated with $1 \mu \mathrm{M}$ of adriamycin (ADR). Following treatment of $0,6,12,24$ and $48 \mathrm{~h}$, $10 \mu \mathrm{l}$ of a modified MTT (Dojindo Laboratories, Kumamoto, Japan) was added to the culture medium and incubated at $37^{\circ} \mathrm{C}$ for $1 \mathrm{~h}$. The absorbance readings for each action were carried out at $570 \mathrm{~nm}$ using the microplate reader (Model 450, Bio-Rad Laboratories, Hercules, CA, USA).

$R N A$ isolation and reverse transcription-polymerase chain reaction ( $R T-P C R)$. The total RNA was prepared from cells using an RNeasy Mini kit (Qiagen, Valencia, CA, USA). The total RNA $(2 \mu \mathrm{g})$ was reverse transcribed using random primers and SuperScript II reverse transcriptase (Invitrogen Life Technologies). The resultant cDNA was subjected to PCR-based amplification. The oligonucleotide primers used were as follows: Forward: 5'-GGGACACTGCCTCATTTCAT-3' and reverse: 5'-CATGGAAGTCCTCCACCTGT-3' for Unc5D; forward: 5'-ATTTGATGCTGTCCCCGGACGATATTGAAC-3' and reverse:5'-ACCCTTTTTGGACTTCAGGTGGCTGGAGTG-3' for p53; forward: 5'-ATGAAATTCACCCCCTTTCC-3' and reverse: 5'-CCCTAGGCTGTGCTCACTTC-3' for P21WAF1; forward: 5'-AGGTGGACCTGTTTCGTGAC-3' and reverse: 5'-ACCCTGTGATCCACCAGAAG-3' for Bax; forward: 5'-ACCTGACCTGCCGTCTAGAA-3' and reverse: 5'-TCCACCACCCTGTTGCTGTA-3' for GAPDH. The PCR products were separated on $2 \%$ gel electrophoresis and visualized by ethidium bromide staining.

Western blot analysis. Cells were extracted directly with lysis buffer containing $25 \mathrm{mM}$ Tris-HCl, $\mathrm{pH} 8.0,137 \mathrm{mM} \mathrm{NaCl}$, $2.7 \mathrm{mM} \mathrm{KCl}, 1 \%$ Triton X-100, $1 \mathrm{mM}$ PMSF and protease inhibitor mixture (Sigma, St. Louis, MO, USA). The total protein concentrations were determined using the Bradford protein assay according to the manufacturer's instructions (Bio-Rad Laboratories). Equal quantities of protein (30-50 $\mu \mathrm{g})$ were boiled for $5 \mathrm{~min}$ in an SDS sample buffer containing $62.5 \mathrm{mM}$ Tris-HCl, pH 6.8, 2\% SDS, $2 \% \beta$-mercaptoethanol and $0.01 \%$ bromophenol blue. Whole cell lysates were separated on $10 \%$ SDS-PAGE and transferred onto a polyvinylidene difluoride membrane (Millipore, Billerica, MA, USA). The membrane was blocked with Tris-buffered saline $[50 \mathrm{mM}$ Tris- $\mathrm{HCl},(\mathrm{pH} 8.0)$, $100 \mathrm{mM} \mathrm{NaCl}$ and $0.1 \%$ Tween-20] containing 5\% non-fat dried milk, and then probed with the monoclonal anti-p53 (Clone Pab1801), monoclonal anti-phosphorylation (Ser-15) p53, goat anti-human Unc5D (R\&D Systems, Minneapolis, MN, USA) or with polyclonal anti-actin (Sigma) antibody. The immunoreactive bands were visualized using horseradish peroxidase-conjugated anti-mouse, anti-rabbit or anti-goat immunoglobulin G antibodies (Jackson ImmunoResearch Laboratories, West Grove, PA, USA) and enhanced chemiluminescence (Amersham Biosciences, Piscataway, NJ, USA). In order to detect the endogenous Unc5D protein, the collected cells were lysed directly in 5X SDS sample buffer [125 mM Tris- $\mathrm{HCl},(\mathrm{pH} 6.8), 4 \%$ SDS, $20 \%$ glycerol, $10 \% \beta$-mercaptoethanol and $0.4 \mathrm{mg} / \mathrm{ml}$ bromophenol blue].
Construction of luciferase reporter plasmids. The indicated luciferase reporter constructs driven by putative p53-responsive elements of the Unc5D gene were generated using the following primers: Forward: 5'-GAGCTCATGTTGGCCAGGCTAGTC-3' and reverse: 5'-GTGCTCACAGGGCAATGACTCACCTC-3' for RE1; and forward: 5'-GGTACCTCACCTCTGAACGTTAAC-3' and reverse: 5'-GGTACCTAAAGGGACTAGATCATG-3' for RE2. The resultant PCR products were gel-purified and inserted into appropriate restriction sites of the pGL3-promoter plasmid (Promega Corporation, Madison, WI, USA), to generate p53-RE1 and p53-RE2, and the constructs were verified by DNA sequencing.

Luciferase reporter assay. The $\mathrm{H} 1299$ cells, which contain deficient p53, were seeded into 12-well cell culture plates. Following overnight culture, the cells were transiently co-transfected with $100 \mathrm{ng}$ pGL3-promoter plasmid (Promega Corporation), p53-RE1 or p53-RE2 and $10 \mathrm{ng}$ plasmids. The total quantity of DNA was kept constant (510 ng) with pcDNA3. At $48 \mathrm{~h}$ after transfection, the cells were lysed and their luciferase activity was measured using the Dual-Luciferase Assay system (Promega Corporation).

Establishment of Unc5D stable clones in 1299 cells. The H1299 cells were transfected with empty plasmid pcDNA3.1 or with the expression plasmid of pcDNA3.1-Unc5D. At $48 \mathrm{~h}$ following transfection, the cells were transfected into the fresh medium containing G418 (Sigma) at a final concentration of $800 \mu \mathrm{g} / \mathrm{ml}$ and incubated for two weeks. Next, G418-resistant clones were selected and cultured in the presence of G418 $(500 \mu \mathrm{g} / \mathrm{ml})$. A total of two mock and two stable clones were selected for future experiments.

Statistical analysis. The data are expressed as the mean \pm standard deviation. A statistical analysis was performed using Student's t-test and $\mathrm{P}<0.05$ was considered to indicate a statistically significant difference.

\section{Results}

Induction of endogenous Unc5D by DNA damage in a p53-dependent manner. In order to examine the effect of Unc5D for cell survival, two neuroblastoma cell lines SH-SY5Y (that contains wild-type p53) and SK-N-BE (which has deficient p53), were tested for cell viability following ADR treatment. SH-SY5Y cells underwent marked cell death compared with SK-N-BE cells (Fig. 1A). DNA damage by ADR treatment clearly induced transcription of Unc5D in SH-SY5Y but not in SK-N-BE cells (Fig. 1B and C). The immunoblot analysis revealed that ADR-mediated apoptosis resulted in an evident induction of endogenous Unc5D. The results indicate that Unc5D was induced in ADR-mediated apoptosis in a p53-dependent manner at the mRNA and protein level, indicating that Unc5D is a target of p53 and also is involved in the DNA damage response.

Unc $5 D$ is a transcriptional target of $p 53$. In order to examine whether Unc5D is a transcriptional target of p53, H1299 cells with deficient p53 were transfected with an expression plasmid encoding p53. Further experiments demonstrated 
A

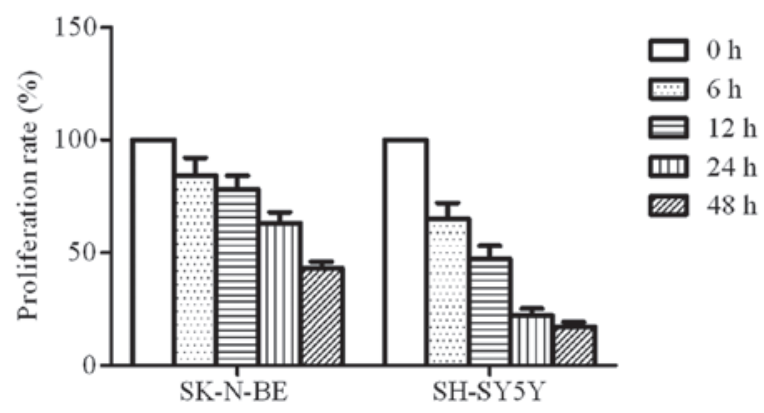

B

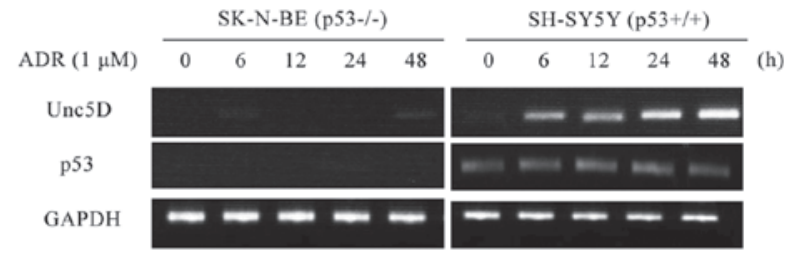

C

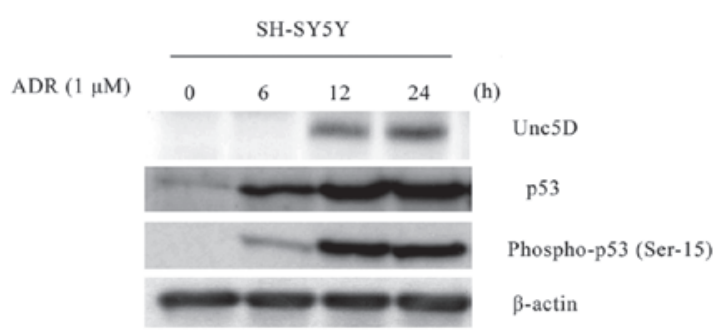

Figure 1. (A) SK-N-BE cells had a higher cell survival rate compared with SH-SY5Y cells following ADR treatment. An 3-(4,5-dimethylthiazol-2-yl)2,5-diphenyltetrazolium bromide solution assay was performed in SK-N-BE $\left(\mathrm{p} 53^{-/}\right)$and SH-SY5Y $\left(\mathrm{p} 53^{+++}\right)$cells subsequent to $1 \mu$ M ADR treatment for the indicated time periods. (B) Unc5D is induced by DNA-damage dependent on the $\mathrm{p} 53$ status. Reverse transcription-polymerase chain reaction was performed in SK-N-BE $\left(\mathrm{p} 53^{--}\right)$and SH-SY5Y $\left(\mathrm{p} 53^{+/+}\right)$cells after $1 \mu \mathrm{M}$ ADR treatment for the indicated time periods. Induction of endogenous Unc5D is dependent upon normal p53 expression. (C) Western blot analysis was used to detect the expression of p53, ser-15 of p53 and Unc5D proteins in SH-SY5Y cell exposure to ADR. The proteins were collected from cells incubated for different periods of time following exposure to stress. Expression of the actin protein was examined as a loading control. ADR, adriamycin.

\section{A}

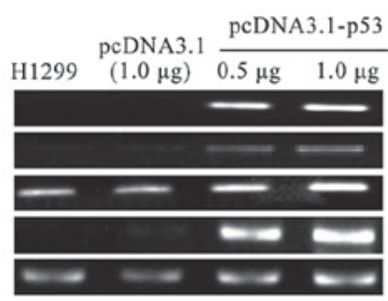

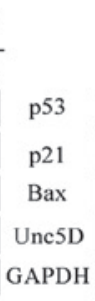

pcDNA3.1-p53

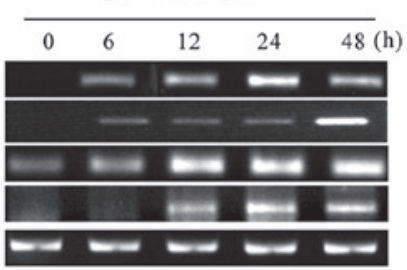

B

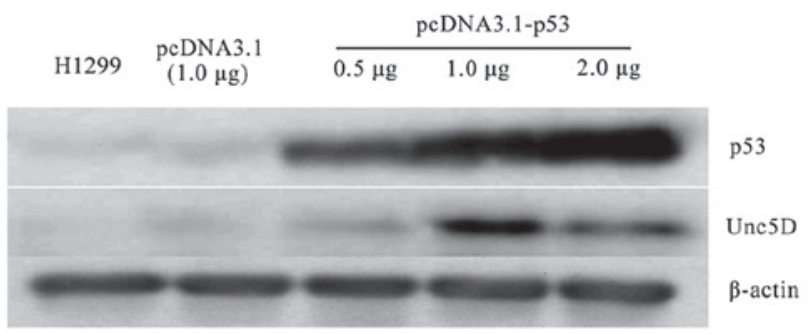

Figure 2. (A) p53 is a direct target of p53. Reverse transcription-polymerase chain reaction was performed in H1299 $\left(\mathrm{p} 53^{--}\right.$) cells following overexpression of $\mathrm{p} 53$ in the indicated time periods and with indicated doses $(0.5$ and $1.0 \mu \mathrm{g})$. Unc5D is induced by the expression of p53, in the same manner as the p53 targets (p21 and Bax). (B) Unc5D is a transcriptional target of p53. Western blot analysis was used to detect the expression of endogenous Unc5D $48 \mathrm{~h}$ after overexpression of p53 with different doses $(0.5,1.0,2.0 \mu \mathrm{g})$ of pcDNA3.1-p53. The p53 deficient cell line H1299 (p53 $\left.3^{-r}\right)$ was used as a negative control of p53. Transfection of an empty vector was used as an experiment control and actin was used as a quantity control.

that p53 induced the expression of Unc5D, p2 $1^{\mathrm{WAF} 1}$ and Bax in a time- and dose-dependent manner (Fig. 2A). Unc5D was induced $12 \mathrm{~h}$ after $\mathrm{p} 53$ transfection, and $6 \mathrm{~h}$ after $\mathrm{p} 53$ expression at the mRNA level (Fig. 2A). Unc5D protein expression was induced in a dose-dependent manner following p53 expression (Fig. 2B). Transfection with the empty plasmid alone did not exhibit a detectable effect on Unc5D, indicating that Unc5D is a direct transcriptional target of p53.

p53 enhances the promoter activity of the Unc5D gene. As Unc5D was induced in the DNA damage response in a p53-dependent manner, it was important to further evaluate the transcription-enhancing activity of the possible p53-responsive elements on the Unc5D gene. The genomic structure of Unc5D and exon-intron organization was presented (Fig. 3A) and two candidate $\mathrm{p} 53$-responsive elements were identified as p53-RE1 and p53-RE2. Cotransfection of p53-RE1 or p53-RE2 with the wide-type p53 expression plasmid significantly increased the luciferase activity compared with the control (Fig. 3B). Therefore, the $\mathrm{p} 53$ responsive elements identified in the present study led to the conclusion that Unc5D is a direct target of p53.

Unc5D is a direct target of p53 and forms a type of feedback to induce apoptosis with DNA damage signal. In the present study, Unc5D induction was observed in SH-SY5Y cells following ADR treatment with an evident accumulation of p53, phosphorylated at ser-15 (Fig. 1C). Furthermore, Unc5D stable-expressing H1299 cells were established. A total of two 
A

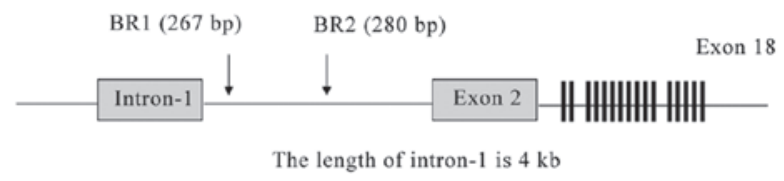

B
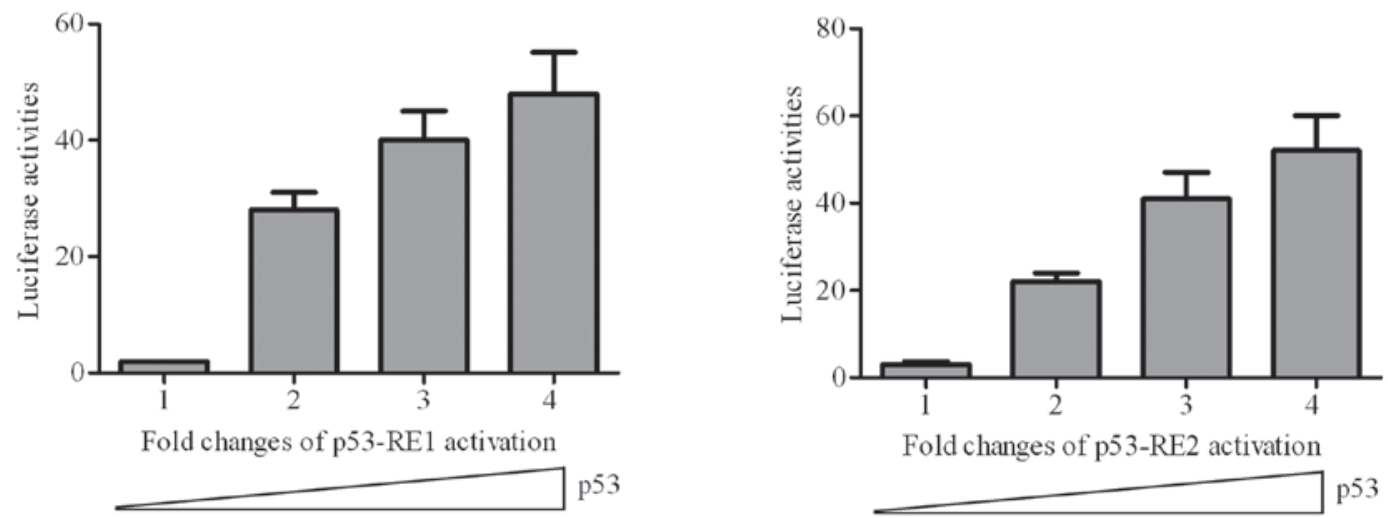

Fold changes of $\mathrm{p} 53-\mathrm{RE} 2$ activation p53

Figure 3. (A) Genomic structure of Unc5D. Exon-intron organization of Unc5D gene was presented. Two potential p53 responsive elements were identified inside intron-1. (B) A luciferase reporter assay shows that p53 enhances the promoter activity of Unc5D gene. H1299 cells were co-transfected with p53 in a dose-dependent manner. The total quantity of plasmid was kept constant by pcDNA3.1. The luciferase activity was normalized based on a Renilla luciferase activity. The graphs indicate the average of three independent experiments.

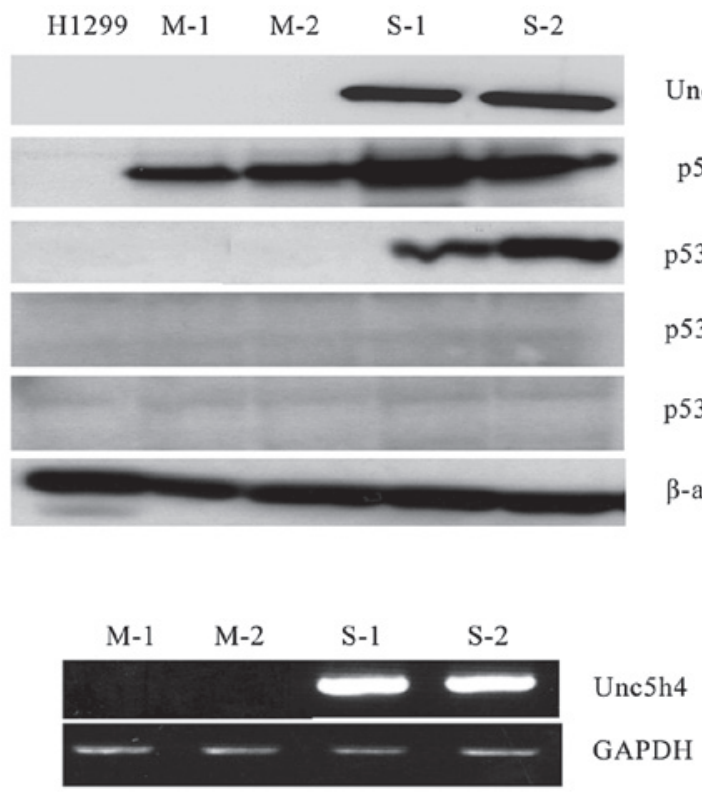

Figure 4. Co-overexpression of Unc5D with p53 causes an increase of p53-Ser15 in H1299 cells. In H1299 cells the Unc5D stable clone 1 and 2 stably expressed Unc5D while the mock stable clones 1 and 2 were not able to. Following transfection with p53, western blot analysis was performed to detect phosphorylation at serine 15 in stable clones but not in the mock clones, as serine- 20 and -46 were undetactable in the two clones. Actin was used as a quantity control.

stable and two mock cell clones were used to detect phosphorylation at several different sites on p53 following DNA damage by ADR. Antibodies that recognized p53 phosphorylation at ser- 15 , ser- 20 and ser-46 were used to detect modified p53 subsequent to ADR treatment and only ser-15 was clearly detectable in Unc5D stable clones compared with that in the

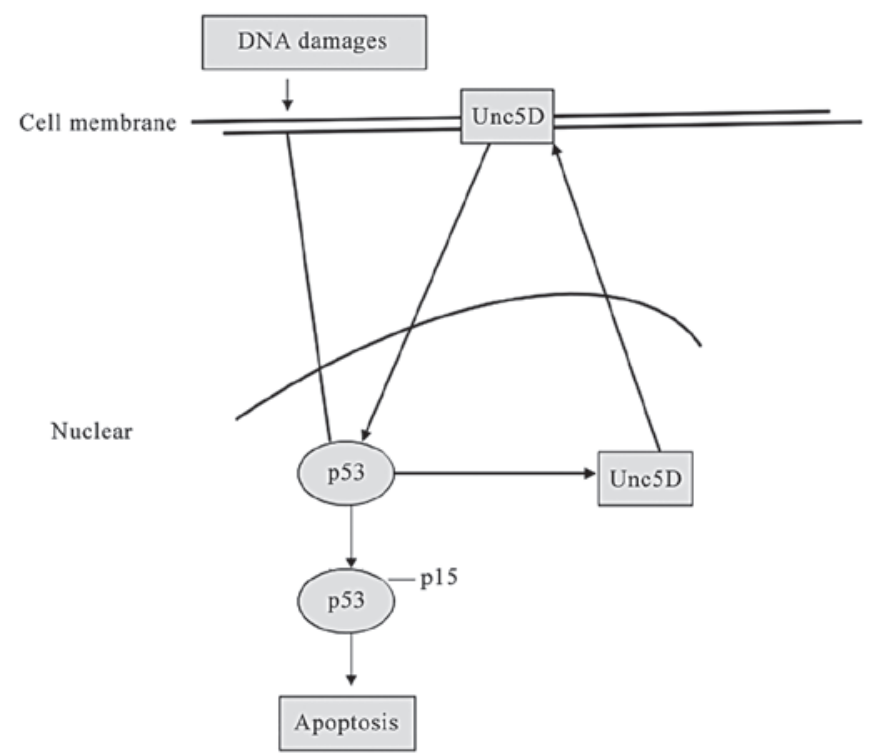

Figure 5. Schematic of how Unc5D expression is induced by p53 following DNA damage and how it activates p53 by phosphorylation at serine-15 as a feedback mechanism.

mock stable clones, of which the other sites were undetectable (Fig. 4). These data reveal that Unc5D is a direct target of p53 following DNA damage, and forms a type of positive feedback to activate p53 by phosphorylation at serine 15 for p53-dependent apoptosis (Fig. 5).

\section{Discussion}

The present study demonstrated that Unc5D was a transcriptional target of p53, and induced apoptosis even in aggressive 
neuroblastoma dependent on the normal p53 status. Unc5D is a member of the Unc5s family, which are the netrin-1 receptors Unc5A, Unc5B, Unc5C and Unc5D. Netrin-1 receptors are a type of transmembrane receptor possibly mediating the chemorepulsive activity during the development of neural cells $(12,13)$, but also promoting cell death induction in the absence of netrin-1, the latter proapoptotic activity depending on the caspase cleavage of these receptors and the conserved death domain located at the C-terminus of their intracellular domains $(14,15)$. Thus they are also termed dependence receptors, which induce cell survival or death dependent on the presence or absent of netrin-1. Unc5B was previously shown to be a direct transcriptional target for the tumor suppressor p53 and to mediate p53 proapoptotic activity (15). In the present study it was shown that Unc5D is significantly induced in DNA damage. Enforced expression of p53 in p53-deficient H1299 cells also induced endogenous Unc5D expression. In SH-SY5Y neuroblastoma cells and in SH-SY5Y p53-proficient cells, Unc5D was induced following adriamycin treatment, following apoptosis in a p53-dependent manner. However, this did not occur in SK-N-BE p53-deficient cells. It remains to be determined how the proapoptotic signal is transmitted from the cell surface to the cytoplasm or nucleus. It is well known that Unc5A-C is cleaved by caspase-3. In addition, a caspase inhibitor or point mutation of the caspase locus can prevent the apoptosis induction (16). However, the specific mechanism downstream from the cell surface, which triggers p53 activation, requires further investigation in order to be clarified.

p53 is a tumor suppressor gene and the most frequent site of genetic alteration found in human cancers. p53 is activated in response to various cellular stresses. The activated p53 binds to specific sequences in the apoptotic-target genes and activates their transcription. Several p53-regulated apoptotic-target genes have been identified and are divided into two major pathways: The mitochondrial and death-receptor pathways. Bax, Noxa and p53 upregulated modulator of apoptosis are involved in the mitochondrial pathway, and Killer/death-inducing receptor 5 and failed axon connections are involved in the death-receptor pathways (17). In the present study, Unc5D is found to be involved in p53-regulated apoptotic pathways, implying the involvement of dependence receptors in a third pathway. Notably Unc5D appears to form a type of feedback to induce p53-dependent apoptosis by transcriptional induction of p53 and phosphorylation at ser- 15 .

Unc5 receptors are involved in vasculogenesis and apoptosis. Unc5B and Unc5D were identified to interact with high-affinity fibronectin leucine rich transmembrane protein 3 (FLRT3) (18). FLRT3 and Unc5B functionally interact in modulating cell adhesion during early Xenopus development, and the effect of Unc5B on adhesion is mediated by the Rho family GTPase 1. Additionally, it has been reported that subventricular expressed transcript 1 (Svet1) contains a high proportion of repetitive sequences and maps in the first intron of Unc5D. The previously reported ' $\mathrm{SVZ}$-specific expression of the Svet1 RNA' indicates putative involvement of Unc5D signaling in the multipolar migrating cells (19). Therefore, certain effects observed in these studies may be due to Svet1, and Svet1 may be upregulated by p53 alone with Unc5D. Additionally, Unc5s can regulate the hepatocyte growth factor/methoprene-tolerant (MET) signaling pathway via an interaction with the intracellular domain of the MET receptor. The MET receptor has a dual anti-apoptotic and pro-apoptotic role in different cell types. While no ligand is bound to MET, the activated MET induces phosphatidylinositol 3-kinase-Akt-dependent signaling leading to the anti-apoptotic response. When no ligand is bound to MET, the receptor is subjected to caspase-dependent cleavage leading to the formation of a pro-apoptotic fragment of MET (20). However, the reason the cells require redundant functions of the different Unc5 proteins, requires further investigation and the identification of other associated proteins in order to elucidate how this transmembrane receptor exerts its cellular functions.

Neuroblastoma treatment is a clinical challenge. Although there have been improvements in chemotherapy, radiotherapy and drug-induced differentiation, even with transplantation, the long-term survival rate of neuroblastoma remains low. Therefore, the identification of novel genes is a prospective way for targeting treatment. Unc5D is a newly identified dependence receptor for netrin-1, and a direct target of p53. Targeting at the Unc5D gene and p53-dependent apoptosis may provide a novel strategy for neuroblastoma treatment.

\section{Acknowledgements}

The authors would like to thank Professor Nakagawara from the Chiba Cancer Center Research Institute, Japan for their technical assistance. This study was supported by a grant from the National Natural Science Foundation of China (grant no. 81101528$)$.

\section{References}

1. Maris JM: Recent advances in neuroblastoma. N Engl J Med 362: 2202-2211, 2010.

2. Hwang SK, Paek SH, Kim DG, Jeon YK, Chi JG and Jung HW: Olfactory neuroblastomas: survival rate and prognostic factor. J Neurooncol 59: 217-226, 2002.

3. Okamura S, Arakawa H, Tanaka T, et al: p53DINP1, a p53-inducible gene, regulates p53-dependent apoptosis. Mol Cell 8: 85-94, 2001.

4. McKenzie PP, Danks MK, Kriwacki RW and Harris LC: P21Waf1/Cip1 dysfunction in neuroblastoma: a novel mechanism of attenuating G0-G1 cell cycle arrest. Cancer Res 63: 3840-3844, 2003.

5. Tanaka $\mathrm{H}$, Arakawa $\mathrm{H}$, Yamaguchi $\mathrm{T}$, et al: A ribonucleotide reductase gene involved in a p53-dependent cell-cycle checkpoint for DNA damage. Nature 404: 42-49, 2000.

6. Yamaguchi T, Matsuda K, Sagiya Y, et al: p53R2-dependent pathway for DNA synthesis in a p53-regulated cell cycle checkpoint. Cancer Res 61: 8256-8262, 2001.

7. Miyashita T and Reed JC: Tumor suppressor p53 is a direct transcriptional activator of the human bax gene. Cell 80: 293-299, 1995.

8. Oda E, Ohki R, Murasawa H, et al: Noxa, a BH3-only member of the Bcl-2 family and candidate mediator of p53-induced apoptosis. Science 288: 1053-1058, 2000.

9. Barak Y, Juven T, Haffner R and Oren M: mdm2 expression is induced by wild type p53 activity. EMBO J 12: 461-468, 1993.

10. Vousden KH: p53: death star. Cell 103: 691-694, 2000.

11. Tanikawa C, Matsuda K, Fukuda S, Nakamura Y and Arakawa H: p53RDL1 regulates p53-dependent apoptosis. Nat Cell Biol 5: 216-223, 2003.

12. Leonardo ED, Hinck L, Masu M, Keino-Masu K, Ackerman SL and Tessier-Lavigne M: Vertebrate homologues of C. elegans UNC-5 are candidate netrin receptors. Nature 386: 833-838, 1997.

13. Hong K, Hinck L, Nishiyama M, Poo MM, Tessier-Lavigne M and Stein E: A ligand-gated association between cytoplasmic domains of UNC5 and DCC family receptors converts netrin-induced growth cone attraction to repulsion. Cell 97: 927-941, 1999. 
14. Llambi F, Causeret F, Bloch-Gallego E and Mehlen P: Netrin-1 acts as a survival factor via its receptors UNC5H and DCC. EMBO J 20: 2715-2722, 2001.

15. Oda K, Arakawa H, Tanaka T, et al: p53AIP1, a potential mediator of p53-dependent apoptosis, and its regulation by Ser-46-phosphorylated p53. Cell 102: 849-862, 2000.

16. Thiebault K, Mazelin L, Pays L, et al: The netrin-1 receptors UNC5H are putative tumor suppressors controlling cell death commitment. Proc Natl Acad Sci USA 100: 4173-4178, 2003.

17. Arakawa H: p53, apoptosis and axon-guidance molecules. Cell Death Differ 12: 1057-1065, 2005.
18. Karaulanov E, Böttcher RT, Stannek P, et al: Unc5B interacts with FLRT3 and Rnd1 to modulate cell adhesion in Xenopus embryos. PLoS One 4: e5742, 2009.

19. Sasaki S, Tabata H, Tachikawa K and Nakajima K: The cortical subventricular zone-specific molecule Svetl is part of the nuclear RNA coded by the putative netrin receptor gene Unc5d and is expressed in multipolar migrating cells. Mol Cell Neurosci 38: 474-483, 2008

20. Kokoszyńska K, Kryński J, Rychlewski L and Wyrwicz LS: Unexpected domain composition of MACC1 links MET signaling and apoptosis. Acta Biochim Pol 56: 317-323, 2009. 